DIGITALCOMMONS - @WAYNESTATE -
Clinical Research in Practice: The Journal of Team Hippocrates

Volume 3 | Issue 1

Article 4

2017

\title{
Combination octreotide, midodrine, and albumin may improve survival in patients with Hepatorenal syndrome, but the evidence is weak
}

Jacob Szafranski

Wayne State University, jszafran@med.wayne.edu

Follow this and additional works at: https://digitalcommons.wayne.edu/crp

Part of the Hepatology Commons, Medical Education Commons, Nephrology Commons, and the Translational Medical Research Commons

\section{Recommended Citation}

Szafranski J. Combination octreotide, midodrine, and albumin may improve survival in patients with hepatorenal syndrome, but the evidence is weak. Clin. Res. Prac. 2017;3(1):eP1136. doi: 10.22237/crp/1484092920

This Critical Analysis is brought to you for free and open access by the Open Access Journals at DigitalCommons@WayneState. It has been accepted for inclusion in Clinical Research in Practice: The Journal of Team Hippocrates by an authorized editor of DigitalCommons@WayneState. 


\title{
Combination octreotide, midodrine, and albumin may improve survival in patients with hepatorenal syndrome, but the evidence is weak
}

JACOB SZAFRANSKI, Wayne State University, Detroit, MI, jszafran@med.wayne.edu

\begin{abstract}
A critical appraisal and clinical application of Skagen C, Einstein M, Lucey MR, Said A. Combination treatment with octreotide, midodrine, and albumin improves survival in patients with type 1 and type 2 hepatorenal syndrome. J Clin Gastroenterol. 2009 Aug;43(7):680-5. doi: 10.1097/MCG.0b013e318188947c
\end{abstract}

Keywords: $\quad$ hepatorenal syndrome, hepatorenal cocktail

\section{Clinical Context}

On the general practice ward, a 52 year old female with a past medical history of primary biliary cirrhosis, presented with left upper quadrant pain. Her MELD score (Model for End Stage Liver Disease) was 22 on presentation, suggesting that she was at high risk of mortality. She was receiving weekly paracentesis, and had been following with hepatology for liver transplantation. For her liver disease, the patient was taking ursodiol, furosemide, spironolactone, lactulose, and rifaximin. After an extensive investigation with physical exam, abdominal ultrasound, esophagogastroduodenoscopy, paracentesis, and chest x-ray, the patient's pain was attributed to musculoskeletal pain of the chest wall. However, during her stay, it was noticed that her creatinine was increasing. Her baseline creatinine prior to presentation was 1.67. During her hospitalization, her creatinine reached a maximum of 2.87 . Working with hepatology and nephrology, we diagnosed hepatorenal syndrome (HRS). Diuretics were discontinued to improve renal perfusion. Nephrology recommended a hepatorenal cocktail composed of octreotide, midodrine, and albumin. The internal medicine staff physician started a conversion with the team about the hepatorenal cocktail. He was not ultimately convinced by the evidence behind the intervention. This inspired me to research hepatorenal syndrome (HRS), intervention options, and the effectiveness of the interventions.

\section{Clinical Question}

Does octreotide, midodrine, and albumin improve survival in patients with hepatorenal syndrome?

JACOB SZAFRANSKI is a student at Wayne State University School of Medicine. 
SZAFRANSKI J. Combination octreotide, midodrine, and albumin may improve survival in patients with hepatorenal syndrome, but the evidence is weak. Clin. Res. Prac. 2017;3(1):eP1136. doi:
Clinical Research in Practice

The Journal of Team Hippocrates

VOL 3 ISS 1 / eP1136 / JANUARY 11, 2017 doi: $10.22237 / \mathrm{crp} / 1484092920$

\section{Research Article}

Skagen C, Einstein M, Lucey MR, Said A. Combination treatment with octreotide, midodrine, and albumin improves survival in patients with type 1 and type 2 hepatorenal syndrome. J Clin Gastroenterol. 2009 Aug;43(7):680-5. doi:

10.1097/MCG.0b013e318188947c

\section{Literature Review}

Hepatorenal syndrome (HRS) is one of many causes of acute kidney injury (AKI) in the context of cirrhosis. Treatment strategies have the goal of reperfusion of the kidneys. I searched PubMed for "hepatorenal syndrome treatment." Many of the most recent papers evaluate terlipressin, an analogue of vasopressin. However, terlipressin is not available in the United States. While initial results are promising $\underline{1}$, it does not help patients and clinicians here and now. One small pilot study $(\mathrm{N}=12)$ looked at the effect of norepinephrine and found norepinephrine effective. $\underline{2}$ One study found that norepinephrine had similar outcomes compared to terlipressin ${ }^{3}$. However, the use of norepinephrine on general medical wards is usually not possible, and therefore was not an option for our patient. A few studies looked at the use of midodrine and octreotide. This therapy was deemed most feasible given our patient's clinical situation. As a search technique, I reviewed the references of a non-systematic consensus review paper published in 2016․ One randomized, double-blinded, cross-over study investigated the effects of octreotide alone as a treatment, but it came to the conclusion that there was no significant improvement in renal function $\stackrel{5}{ }$. This paper was underpowered because there were only 14 cases. One retrospective study compared octreotide/midodrine to dopamine. It showed a significant positive outcome with octreotide/midodrine, but the study only had 13 participants ${ }^{-}$. Another retrospective study used octreotide, midodrine, and albumin, all of which are generally options to a patient on the general medical wards. The study had 81 participants. The study also claimed to have a significant outcome for patients with hepatorenal syndrome but the methodology was weak for an observational trial ${ }^{7}$. The study selected for this appraisal showed a significant outcome with the use of octreotide, midodrine, and albumin, similar to the previous study, but had a total of 162 participants $\stackrel{8}{\text {. }}$

\section{Critical Appraisal}

The paper is a retrospective study from a single hospital between the years 1997-2004. Billing database information was used for case finding using ICD-9 codes. The authors gave the impression that they included all patients who received the specified treatment, although the methodology for case finding was only as good as was achievable using their retrospective methods. Historical controls were identified using the same methods, and this group of patients were a subset of patients prior to availability of the therapy studied. The study has a $3 \mathrm{~b}$ level of evidence using the Oxford Centre for Evidence-Based Medicine. Selected participants were identified to have HRS. The authors used reasonable inclusion and exclusion criteria in what seemed to be a consistent manner. Our patient would have been eligible for inclusion. There were 75 in the treatment group who received octreotide, midodrine, and albumin, and 87 in the control group, for a total of 162 cases. Both groups were similar in regard to MELD score, baseline GFR, baseline creatinine, liver disease etiology, and severity of HRS (HRS 1 and 2). Although a handful of objective measurements are similar between both groups, other confounding factors that were different between the groups cannot be ascertained. These confounding variables may be responsible for the end results of the study. Once the treatment was available, the study does not include those that did not receive treatment in the statistical analysis. Those that did not receive the treatment might not have received it for a variety of reasons, creating a bias between the groups. This is perhaps the critical flaw in the paper. The authors did not compare patients who received the therapy to patients who did not receive the therapy once it became available. This limits our ability to assess the bias. These ignored individuals after May 2001 were inevitably present in the pre-May 2001 group and therefore included in the control group, another source of bias. Due to the retrospective nature of this paper, the treatment groups were not randomized and were not blinded. No inference of cause and effect can be made using this study methodology. In light of this, the reader must be cautious during clinical application.

All-cause mortality is possible to determine in a retrospective study, given the ability to determine living status with social security records. However, morbidity is a more difficult task. The other outcomes this study looked into besides mortality were glomerular filtration rate (GFR), liver transplant rates, and dialysis. There are many other measurements of outcome that could be determined and could influence the decision to use treatment. 
SZAFRANSKI J. Combination octreotide, midodrine, and albumin may improve survival in patients with hepatorenal syndrome, but the evidence is weak. Clin. Res. Prac. 2017;3(1):eP1136. doi: $10.22237 / \mathrm{crp} / 1484092920$
Clinical Research in Practice The Journal of Team Hippocrates

VOL 3 ISS 1 / eP1136 / JANUARY 11, 2017 doi: $10.22237 / \mathrm{crp} / 1484092920$

The overall survival between the treatment group and control group at 3 months was $53 \%$ vs. $27 \%$ ( $p<0.0001$ ). The median survival between the groups is 101 days vs. 18 days, respectively. This has the appearance of a very large effect size, but again, caution should be used when drawing conclusions. Mortality as the outcome is less likely to be a source of ascertation bias. The number needed to treat with midodrine-octreotide-albumin compared to standard care over a three month period of time is calculated to be 3.8. The study also calculated the survival rates between individuals with HRS 1 and HRS 2. HRS 1 three-month survival was $44 \%$ vs. $18 \%(p<0.0004)$, number needed to treat being 3.8. HRS 2 three-month survival was $75 \%$ vs $40 \%(p<0.007)$, number needed to treat being 2.9. All $p$ values indicate statistically significant results.

\section{Clinical Application}

The gold standard for a patient with liver cirrhosis is liver transplantation, especially if he/she is burdened by hepatorenal syndrome. The scarcity of resources, however, requires us as physicians to develop alternative treatment options. This study by Skagen, et al. shows us that the use of octreotide, midodrine, and albumin have a positive outcome on survival rates for patients with hepatorenal syndrome. As a retrospective study, this paper provides only modest guidance to clinical application. Telling a patient that there is improved survival sounds much different than telling him/her that median survival increases from 18 to 101 days; the difference is even greater when explaining we must treat 3.8 people to see improvement in 1 . This is where the discussion of side effects and number needed to harm comes into play, but that is beyond the scope of this paper and the study appraised. Ultimately, the patient has the right to decide if the outcome is significant within their life context.

For our patient, it was reasonable for the patient and clinicians to initiate treatment with midodrine, octreotide, and albumin (HRS cocktail). Our patient had many similar features compared to the patients in the study: similar age, MELD score, race. One example of how our patient differed is that the majority of the patients in the study had liver disease due to alcohol, hepatitis $C$, and hepatitis $B$, while our patient's was due to primary biliary cirrhosis. Ultimately, based on our pathophysiologic understanding of this disease, it is reasonable to say that our patient was similar to the patients of this study. Improved survival is noted at 3 months. This gives our a greater chance to receive a liver for transplantation, which is the ultimate treatment for hepatorenal syndrome.

In other situations, however, improved survival may not be valued as much. Another patient comes to mind, who also had end-stage liver cirrhosis, but did not meet inclusion criteria for liver transplantation. If he were to be faced with the clinical situation of HRS, how would he value the statistical numbers of improved survival? I cannot speak for him, but it was apparent after many early-morning discussions that he wanted to leave the hospital as soon as possible so that he could spend his statistically-limited days with his family. Also, as mentioned earlier as a limitation of this retrospective study, strong analysis of morbidity is difficult. Is there potentially a side effect of the HRS cocktail that may impair his final days with his family? In this case of no transplantation option the interpretation of improved survival changes, and can only be made on a case-by-case basis.

Although HRS cocktail may be appropriate in some patients, it is also reasonable to forego such therapy, given the lack of any strong evidence of its efficacy.

Learning points

1.) Midodrine, Octreotide, and Albumin may improve survival for patients with hepatorenal syndrome at 3 months, improving chances to be selected for liver transplantation, if eligible.

2.) Retrospective studies have limitations. Given the overall paucity of studies on this therapy despite lack of other treatments, one has to wonder if the benefits are convincing enough to initiate a randomized doubleblinded controlled trial.

3.) Terlipressin has been shown to be superior to the HRS cocktail, but it is not yet available in our practice.

\section{References}

1. Cavallin M, Kamath PS, Merli M, et al.,Terlipressin plus albumin versus midodrine and octreotide plus albumin in the treatment of hepatorenal syndrome: A randomized trial. Hepatology. 2015 Aug;62(2):567-74. doi: 10.1002/hep.27709 
SZAFRANSKI J. Combination octreotide, midodrine, and albumin may improve survival in patients with hepatorenal syndrome, but the evidence is weak. Clin. Res. Prac. 2017;3(1):eP1136. doi:

$\underline{10.22237 / \mathrm{crp} / 1484092920}$
Clinical Research in Practice The Journal of Team Hippocrates

VOL 3 ISS 1 / eP1136 / JANUARY 11, 2017 doi: $10.22237 / \mathrm{crp} / 1484092920$

2. Duvoux C, Zanditenas D, Hézode C, et al. Effects of noradrenalin and albumin in patients with type I hepatorenal syndrome: a pilot study. Hepatology. 2002;36:374-380 doi: 10.1053/ihep.2002.34343

3. Ghosh S, Choudhary NS, Sharma AK, et al. Noradrenaline vs terlipressin in the treatment of type 2 hepatorenal syndrome: a randomized pilot study. Liver Int. 2013 Sep;33(8):1187-93. doi: 10.1111/liv.12179

4. Baraldi O, Valentini C, Donati G, et al. Hepatorenal syndrome: Update on diagnosis and treatment. World Journal of Nephrology. 2015;4(5):511-520. doi: 10.5527/wjn.v4.i5.511.

5. Pomier-Layrargues G. Octreotide in hepatorenal syndrome: A randomized, double-blind, placebo-controlled, crossover study. Hepatology. 2003;38(1):238-243. doi: 10.1053/jhep.2003.50276

6. Angeli P, Volpin R, Gerunda G, et.al., Reversal of type 1 hepatorenal syndrome with the administration of midodrine and octreotide. Hepatology. 1999 Jun;29(6):1690-7. doi: 10.1002/hep.510290629

7. Esrailian E, Pantangco R, Kyulo NL, et.al., Octreotide/Midodrine therapy significantly improves renal function and 30-day survival in patients with type 1 hepatorenal syndrome. Dig Dis Sci. 2007 Mar;52(3):742-8. doi: 10.1007/s10620-006-9312-0

8. Skagen C, Einstein M, Lucey MR, Said A., Combination treatment with octreotide, midodrine, and albumin improves survival in patients with type 1 and type 2 hepatorenal syndrome. J Clin Gastroenterol. 2009 Aug;43(7):680-5. doi:

10.1097/MCG.0b013e318188947c 